International Journal of Modern Physics A, (C) World Scientific Publishing Company

\title{
GEOMETRIES OF ORTHOGONAL GROUPS AND THEIR CONTRACTIONS: A UNIFIED CLASSICAL DEFORMATION VIEWPOINT
}

\author{
M. SANTANDER \\ Departamento de Física Teórica, Universidad de Valladolid \\ E-47011 Valladolid, Spain \\ F. J. HERRANZ \\ Departamento de Física, E.U. Politécnica Universidad de Burgos \\ E-09006, Burgos, Spain
}

\begin{abstract}
The geometries of spaces having as groups the real orthogonal groups and some of their contractions are described from a common point of view. Their central extensions and Casimirs are explicitly given. An approach to the trigonometry of their spaces is also advanced.
\end{abstract}

\section{Aim and Outline}

The general aim of this paper is to describe a particular case of a classical scheme which involves a whole class of spaces, and geometries associated to a family of Lie groups. At all different levels of this scheme, either the spaces, the Lie groups or their Lie algebras are related among themselves by contractions, yet their properties can be dealt with in a completely unified way. The family we will consider here comprises the classical real geometries of spaces with a projective metric (Cayley-Klein or CK geometries) 1 , together with their Lie groups and Lie algebras. These algebras will be called here orthogonal CK algebras, as they include all simple orthogonal real Lie algebras $s o(p, q)$, as well as many Lie algebras of great physical relevance, (Poincaré, Galilei, Euclidean, etc) which are obtained by different contractions from $s o(p, q)$. All two-point homogeneous symmetric spaces of real type (the Riemannian spaces of constant curvature) appear related to CK algebras, but pseudoRiemannian and degenerate Riemannian spaces (which strictu senso are not two-point homogeneous) are included as well; indeed this class of spaces is more natural than the class of two-point homogeneous spaces as usually considered in the literature.

The orthogonal CK scheme is one of the several (though in reduced number) possible schemes of a similar kind. Each include some simple real Lie algebras, as well as some non-simple contracted algebras, which are however 'near' to the simple ones so that most properties of simple algebras (geometries, groups), when suitably reformulated, still survive for them; this makes 'quasi-simple' an apt name 
for these algebras $\mathrm{B}$. The algebras we will study here can therefore be called also 'quasi-orthogonal'; a further reason to study first this family is that all other families always include a orthogonal CK subalgebra.

All orthogonal CK algebras allow many of its properties to be simultaneouly studied. As two recent examples, we give here the general result for the second cohomology group of CK algebras (Section 3) and general expressions for all their Casimir operators (Section 4). The study of the geometry of homogeneous spaces associated to CK algebras, sketched in Section 5, is the paradigm of this simultaneous approach. The paper is meant as an overview of the CK approach, including results and topics under current development. Another part of this project, which deals with expansions (somehow the opposite proccess to contractions) is covered in another paper in this volume 3 .

\section{The orthogonal CK family}

Let $\omega_{1}, \ldots, \omega_{N}$ be $N$ real numbers. For $a, b=0,1, \ldots, N, a<b$, let us denote $\omega_{a b}:=\omega_{a+1} \omega_{a+2} \ldots \omega_{b}=\prod_{i=a+1}^{b} \omega_{i}$ (thus, $\omega_{a b} \omega_{b c}=\omega_{a c}$ ), and consider a family (parametrized by the constants $\omega_{i}$ ) of real Lie algebras with $N(N+1) / 2$ generators $\Omega_{a b}, a<b$, whose Lie brackets are given by

$$
\left[\Omega_{a b}, \Omega_{a c}\right]=\omega_{a b} \Omega_{b c}, \quad\left[\Omega_{a b}, \Omega_{b c}\right]=-\Omega_{a c}, \quad\left[\Omega_{a c}, \Omega_{b c}\right]=\omega_{b c} \Omega_{a b}, \quad a<b<c,
$$

and commutators involving four different indices are zero. These algebras are called orthogonal CK Lie algebras (or quasi-orthogonal algebras) and appear as a natural particular subfamily of all the graded contractions from the Lie algebra $s o(N+1)$ 4 corresponding to a $\mathbb{Z}_{2}^{\otimes N}$ grading of $s o(N+1)$; this grading is related to a set of $N$ commuting involutive automorphisms $I_{(1)}, I_{(2)}, \ldots I_{(N)}$ of the algebra (1). A natural labelling for these algebras is $s o_{\omega_{1}, \omega_{2}, \ldots, \omega_{N}}(N+1)$, a notation which makes explicit the parameters $\omega_{i}$ and generalizes the standard $s o(N+1) \equiv s o_{1,1, \ldots, 1}(N+1)$. The CK algebras have a (vector) representation by $(N+1) \times(N+1)$ real matrices: $\Omega_{a b}=-\omega_{a b} e_{a b}+e_{b a}$ where $e_{a b}$ is the matrix with a single non-zero entry, 1 , in the row $a$, column $b$. Since each coefficient $\omega_{i}$ may take positive, negative or zero values, and by means of a simple rescaling of the initial generators can be reduced to the standard values of $1,-1$ or 0 , it is clear that the family $s_{\omega_{1}, \ldots, \omega_{N}}(N+1)$ includes $3^{N}$ Lie algebras, which are different as graded contractions, even if two of them can still be isomorphic.

When all the $\omega_{i}$ are non-zero but some of them are negative, each algebra is isomorphic to a certain pseudo-orthogonal algebra $s o(p, q)(p+q=N+1, p \geq$ $q>0$ ). When however a given constant $\omega_{i}=0$, the algebra has a semidirect structure. These are obtained from $s o(p, q)$ through a sequence of Inönü-Wigner (IW) contractions, and a distinctive trait of the CK scheme is the ability to describe the resulting contracted algebras by simply setting a constant equal to zero, $\omega_{i}=$ 0 . When several constants $\omega_{i}$ are equal to zero, the resulting algebra has several semidirect splittings (and hence several inhomogeneous structures) into an abelian subalgebra and an eventual direct sum of CK algebras in lower dimensions. 
For any $m,(m=1, \ldots, N)$, let $\mathfrak{h}^{(m)}$ denote the subalgebra generated by those $\Omega_{a b}$ where the indices $a, b$ satisfy either $b<m$ or $a \geq m$. A complement for $\mathfrak{h}^{(m)}$ is the vector subspace $\mathfrak{p}^{(m)}$ (not always a subalgebra) spanned by the elements $\Omega_{a b}$ with $a<m$ and $b \geq m$. The structure of the subalgebras $\mathfrak{h}^{(m)}$ and of the vector subspace $\mathfrak{p}^{(m)}$ of the Lie algebra $s o_{\omega_{1}, \omega_{2}, \ldots, \omega_{N}}(N+1)$ can be graphically displayed by arranging the generators of $s o(N+1)$ in the form of a triangle

$$
\begin{aligned}
& \begin{array}{llll|llll}
\Omega_{01} & \Omega_{02} & \ldots & \Omega_{0 m-1} & \Omega_{0 m} & \Omega_{0 m+1} & \ldots & \Omega_{0 N}
\end{array} \\
& \begin{array}{lll|llll}
\Omega_{12} & \ldots & \Omega_{1 m-1} & \Omega_{1 m} & \Omega_{1 m+1} & \ldots & \Omega_{1 N}
\end{array} \\
& \begin{array}{cc|cccc}
\ddots & \vdots & \vdots & \vdots & & \vdots
\end{array} \\
& \begin{array}{l|llll}
\Omega_{m-2 m-1} & \Omega_{m-2 m} & \Omega_{m-2 m+1} & \ldots & \Omega_{m-2 N}
\end{array} \\
& \begin{array}{cccc}
\Omega_{m-1 m} & \Omega_{m-1 m+1} & \ldots & \Omega_{m-1 N} \\
\hline \Omega_{m m+1} & \ldots & \Omega_{m N}
\end{array} \\
& \ddots \quad \vdots \\
& \Omega_{N-1 N}
\end{aligned}
$$

We see that the subspace $\mathfrak{p}^{(m)}$ is spanned by those $m(N+1-m)$ generators in the rectangle whose bottom left corner is $\Omega_{m-1 m}$, the left and down triangles corresponding respectively to the subalgebras $s o_{\omega_{1} \ldots \omega_{m-1}}(m)$ and $s o_{\omega_{m+1} \ldots \omega_{N}}(N+1-m)$ respectively, whose direct sum is the subalgebra $\mathfrak{h}^{(m)}$. In fact the action of the involutions $I_{(m)}$ on the generators have $\mathfrak{p}^{(m)}$ and $\mathfrak{h}^{(m)}$ as the antiinvariant and invariant subspaces, and the decomposition $s o_{\omega_{1}, \omega_{2}, \ldots, \omega_{N}}(N+1)=\mathfrak{p}^{(m)} \oplus \mathfrak{h}^{(m)}$ is a Cartan-like decomposition, each of which has an associated IW contraction, defined as the $\epsilon \rightarrow 0$ limit of the CK algebra transformation:

$$
\mathbb{X} \mapsto \Gamma^{(m)}(\mathbb{X})=\left\{\begin{array}{rll}
\mathbb{X} & \text { if } & \mathbb{X} \in \mathfrak{h}^{(m)} \\
\epsilon \mathbb{X} & \text { if } & \mathbb{X} \in \mathfrak{p}^{(m)}
\end{array} \quad m=1, \ldots, N .\right.
$$

It is clear that under the contraction $\Gamma^{(m)}$, the algebra $s o_{\omega_{1}, \omega_{2}, \ldots, \omega_{N}}(N+1)$ goes to another algebra in the CK family, with the same values of the $\omega_{i}$ constants except for the new $\omega_{m}=0$. In the triangular arrangement of generators, the generators in the rectangle are abelianized, while commutators where at least a generator is outside this rectangle remain unchanged.

\section{Central extensions}

Any central extension $\overline{s o}_{\omega_{1}, \ldots, \omega_{N}}(N+1)$ of the algebra $s o_{\omega_{1}, \ldots, \omega_{N}}(N+1)$ by the onedimensional algebra of generator $\Xi$ will have generators $\left(\Omega_{a b}, \Xi\right)$ and commutators:

$$
\left[\Omega_{a b}, \Omega_{c d}\right]=\sum_{i, j=0}^{N} C_{i<j}^{i j} C_{a b, c d} \Omega_{i j}+\alpha_{a b, c d} \Xi, \quad\left[\Xi, \Omega_{a b}\right]=0,
$$

where $C_{a b, c d}^{i j}$ are those in eq. (1). The extension is determined by a two-cocycle (whose components in the $\Omega_{a b}$ basis are $\alpha_{a b, c d}$ ), and two-cocycles differing by a two-coboundary correspond to equivalent extensions, which are therefore classified 
by the second cohomology group of the algebra. The problem of determining the central extensions of the algebra (11) has therefore two stages. First, find the possible cocycles (the two-cocycle condition reducing to the requirement that (3) is a Lie algebra). Second, determine when a two-cocycle is trivial (i.e., classify these into cohomology classes).

The general solution of these two problems can be stated in the following 6 :

Theorem 1: The independent non-zero commutators of any central extension $\overline{s o}_{\omega_{1}, \ldots, \omega_{N}}(N+1)$ of the CK Lie algebra $s_{\omega_{1}, \ldots, \omega_{N}}(N+1)$ can be written as:

$$
\begin{array}{lll}
{\left[\Omega_{a b}, \Omega_{b c}\right]=-\Omega_{a c}} & & \\
{\left[\Omega_{a b}, \Omega_{a b+1}\right]=\omega_{a b} \Omega_{b b+1}+\omega_{a b-1} \alpha_{b b+1}^{F} \Xi} & {\left[\Omega_{a b}, \Omega_{a c}\right]=\omega_{a b} \Omega_{b c}} & \text { for } c>b+1 \\
{\left[\Omega_{a c}, \Omega_{a+1 c}\right]=\omega_{a+1} \Omega_{a a+1}+\omega_{a+2 c} \alpha_{a+1}^{L}} & \left.\Xi \Omega_{a c}, \Omega_{b c}\right]=\omega_{b c} \Omega_{a b} & \text { for } b>a+1 \\
{\left[\Omega_{a a+1}, \Omega_{c c+1}\right]=\beta_{a+1 c+1} \Xi} & {\left[\Omega_{a a+2}, \Omega_{a+1 a+3}\right]=-\omega_{a+2} \beta_{a+1 a+3} \Xi}
\end{array}
$$

where any $\omega_{a a}$ with two equal indices should be understood as $\omega_{a a}:=1$. The extension is completely described by a number of extension coefficients:

- Two single coefficients, $\alpha_{01}^{L}, \alpha_{N-1 N}^{F}$. The extension $\alpha_{01}^{L}$ (resp. $\alpha_{N-1 N}^{F}$ ) is nontrivial if $\omega_{2}=0\left(\right.$ resp $\left.\omega_{N-1}=0\right)$ and is trivial otherwise.

- $(N-2)$ pairs, $\alpha_{12}^{F}, \alpha_{12}^{L}, \ldots, \alpha_{N-2 N-1}^{F}, \alpha_{N-2 N-1}^{L}$. Each pair of coefficients must satisfy $\omega_{a+3} \alpha_{a+1 a+2}^{F}=\omega_{a+1} \alpha_{a+1 a+2}^{L}$. The two extensions corresponding to the pair $\alpha_{a+1 a+2}^{F}$ and $\alpha_{a+1 a+2}^{L}$ are both non-trivial when $\omega_{a+1}=0$ and $\omega_{a+3}=0$. The two two-cocycles are simultaneously trivial otherwise.

- $(N-2)$ extension coefficients $\beta_{13}, \beta_{24}, \ldots, \beta_{N-2 N}$. The coefficient $\beta_{b+1 b+3}$ satisfies $\omega \beta_{b+1 b+3}=0$ for $\omega=\omega_{b}, \omega_{b+1} \omega_{b+2}, \omega_{b+2} \omega_{b+3}, \omega_{b+4}$ where when either $b=0$ or $b=N-3$ the first or last conditions, which would read $\omega_{0} \beta=0$ or $\omega_{N+1} \beta=0$ are not present. The extension corresponding to any of these non-zero coefficients is always non-trivial.

- $(N-2)(N-3) / 2$ extension coefficients $\beta_{14}, \beta_{15}, \ldots, \beta_{1 N}, \beta_{25}, \ldots, \beta_{2 N}, \ldots, \beta_{N-3 N}$ whose indices differ by more than two. The coefficient $\beta_{b+1 d+1}$ satisfies $\omega \beta_{b+1 d+1}=$ 0 for $\omega=\omega_{b}, \omega_{b+2}, \omega_{d}, \omega_{d+2}$ with similar restrictions as to the actual presence of the equations involving the non-existent constants $\omega_{0}$ or $\omega_{N+1}$. The extension corresponding to any of these non-zero coefficients is always non-trivial.

\section{Casimir Operators}

When all $\omega_{i}$ are different from zero, $s o_{\omega_{1}, \ldots, \omega_{N}}(N+1)$ is a simple algebra, whose rank is $l=\left[\frac{N+1}{2}\right]$. The dimension of the center of its universal enveloping algebra equals the rank $l$ of the algebra, and is generated by a set of homogeneous polynomials (Casimir operators) of orders $2,4, \ldots,\left[\frac{N}{2}\right]$, and an additional Casimir of order $l$ when $N+1$ is even. Upon any contraction $\omega_{m} \rightarrow 0$, and after a suitable rescaling, these Casimirs go into non-trivial Casimir operators for the contracted algebra. Furthermore, these are a complete set of Casimirs for CK algebras. This provides another justification for the name 'quasi-simple' given to its members.

We now givel the expressions for the $\left[\frac{N+1}{2}\right]$ Casimir operators in the general CK 
Lie algebra $s o_{\omega_{1}, \ldots, \omega_{N}}(N+1)$. We do this by using an approach where the behaviour of Casimirs under the family of contractions $\omega_{m} \rightarrow 0$ is transparent.

Starting from the generators of the Lie algebra $s \omega_{\omega_{1}, \ldots, \omega_{N}}(N+1)$, we define some elements in its universal enveloping algebra, $W_{a b}, W_{a_{1} a_{2} b_{1} b_{2}}, \ldots$ The recursive definition starts from $W_{a b}:=\Omega_{a b},(a<b)$ and further $W$ symbols with four indices $W_{a_{1} a_{2} b_{1} b_{2}}$ (where $a_{1}<a_{2}<b_{1}<b_{2}$ ), six indices $\ldots$, until symbols with $2 l$ indices $W_{a_{1} a_{2} \ldots a_{l} b_{1} b_{2} \ldots b_{l}}$ (with $a_{1}<a_{2}<\ldots<a_{l}<b_{1}<b_{2}<\ldots<b_{l}$ ), are given in terms of those with two less indices. For $s=2, \ldots, l$ the $2 s$-index symbol $W$ is given in terms of the $(2 s-2)$-index symbol $W$ by means of:

$$
\begin{aligned}
W_{a_{1} a_{2} \ldots a_{s} b_{1} b_{2} \ldots b_{s}}= & \sum_{\mu=1}^{s}(-1)^{\mu} \Omega_{a_{\mu} b_{s}} W_{\left\{a_{1} a_{2} \ldots a_{s} b_{1} b_{2} \ldots b_{s}\right\}-\left\{a_{\mu} b_{s}\right\}} \\
& +\sum_{\nu=1}^{s-1}(-1)^{s+\nu} \omega_{a_{s} b_{\nu}} \Omega_{b_{\nu} b_{s}} W_{\left\{a_{1} a_{2} \ldots a_{s} b_{1} b_{2} \ldots b_{s}\right\}-\left\{b_{\nu} b_{s}\right\}} .
\end{aligned}
$$

where the $W$ symbols in the right hand side of the equation have $2 s-2$ indices, those obtained by removing the two indices $\left\{a_{\mu} b_{s}\right\}$ from the set of $2 s$ indices $\left\{a_{1} a_{2} \ldots a_{s} b_{1} b_{2} \ldots b_{s}\right\}$. In terms of these symbols, the Casimirs are given by:

Theorem 2: The $l=\left[\frac{N+1}{2}\right]$ independent polynomial Casimir operators of the CK Lie algebra $s o_{\omega_{1}, \ldots, \omega_{N}}(N+1)$ can be written as:

- $\left[\frac{N}{2}\right]$ invariants $\mathcal{C}_{s}, s=1, \ldots,\left[\frac{N}{2}\right]$ of order $2 s$ :

$$
\mathcal{C}_{s}=\sum_{\substack{a_{1} a_{2} \ldots a_{s} b_{1} b_{2} \ldots b_{s}=0 \\ a_{1}<a_{2}<\ldots<a_{s}<b_{1}<b_{2}<\ldots<b_{s}}}^{N} \omega_{0 a_{1}} \omega_{1 a_{2}} \ldots \omega_{s-1 a_{s}} \omega_{b_{1} N-s+1} \omega_{b_{2} N-s+2} \ldots \omega_{b_{s} N} W_{a_{1} a_{2} \ldots a_{s} b_{1} b_{2} \ldots b_{s}}^{2}
$$

- When $N+1$ is even, there is an extra Casimir $\mathcal{C}$ of order $l=\frac{N+1}{2}, \mathcal{C}=W_{012 \ldots N}$.

Again in these expresions any $\omega_{a a}$ should be understood as $\omega_{a a}:=1$. It is easy to see that even in the most contracted CK algebra, the flag space algebra, $s o_{0, \ldots, 0}(N+1)$, these Casimirs are not trivial. In fact, the term in eq. (6) with the $W$ symbol whose first group of $s$ indices are consecutive and start from 0 , and whose last group of $s$ indices are also consecutive and end with $N, W_{012 \ldots s-1 N-s+1 \ldots N-2 N-1 N}^{2}$ is the only term whose $\omega$ factor is equal to 1 , and therefore the only which survives in the Casimir $\mathcal{C}_{s}$ for the $s o_{0, \ldots, 0}(N+1)$ algebra.

\section{The CK scheme of homogeneous spaces}

An aggregate of $N$ homogeneous symmetric spaces can be allocated to each algebra $s o_{\omega_{1}, \omega_{2}, \ldots, \omega_{N}}(N+1)$. If the group obtained by exponentiation of the generators $\Omega_{a b}$ is naturally denoted as $S O_{\omega_{1}, \omega_{2}, \ldots, \omega_{N}}(N+1)$, then the subgroup generated by $\mathfrak{h}^{(m)}$ is easily shown to be $S O_{\omega_{1}, \ldots, \omega_{m-1}}(m) \otimes S O_{\omega_{m+1}, \ldots, \omega_{N}}(N+1-m)$. Therefore, for each $m=1, \ldots, N$ we can construct the coset space $\mathcal{S}^{(m)}:=S O_{\omega_{1}, \omega_{2}, \ldots, \omega_{N}}(N+$ 1)/[SO $\left.O_{\omega_{1}, \ldots, \omega_{m-1}}(m) \otimes S O_{\omega_{m+1}, \ldots, \omega_{N}}(N+1-m)\right]$, of dimension $m(N+1-m)$, which 
is a homogeneous space for the CK group $S O_{\omega_{1}, \omega_{2}, \ldots, \omega_{N}}(N+1)$. Furthermore, the $N$ commuting involutive automorphisms $I_{(1)} \ldots I_{(N)}$ endow these coset spaces with a structure of symmetric space, the involution $I_{(m)}$ playing the role of geodesic reflection around the origin in $\mathcal{S}^{(m)}$. Of course, all these homogeneous spaces are built from the same Lie algebra/group, with a unified procedure, and they are interlinked, as each space can be interpreted in terms of any other. This is in tune with a 'Hilbert-like' approach, where points lines, planes, ..., appear with the same footing and no preponderance is given to any of them above others.

In terms of the triangular arrangement of generators, the $N$ possible homogeneous spaces associated to the CK $s_{\omega_{1}, \omega_{2}, \ldots, \omega_{N}}(N+1)$ algebra correspond to the $N$ different rectangles that can be selected inside the large triangle. The generators in this rectangle generate translations along lines through the origin of the space, and the subspace $\mathfrak{p}^{(m)}$ is identified to the tangent space to $\mathcal{S}^{(m)}$ at the origin.

Given two different points in $\mathcal{S}^{(m)}$, it can be shown that the number of functionally independent invariants under the group action is the minimum of the two values $m, N+1-m$. This number will be called here the rank of the CK homogeneous space. When all constants $\omega_{i}$ are positive (and therefore can be asumed to be equal to 1$)$, the homogeneous space is exactly the Grassmannian $G(m, N+1-m) \equiv S O(N+1) /\left(S O(m) \otimes S O(N+1-m)\right.$ of $m$-planes in the $\mathbb{R}^{N+1}$ ambient space; for this case (in general, whenever all $\omega_{i} \neq 0$ ), the rank of the space also coincides with the maximal dimension of a totally geodesic and flat submanifold.

\subsection{CK geometries}

Each CK symmetric homogeneous space $\mathcal{S}^{(m)}$ inherits from its algebra/group:

- an invariant canonical connection, associated to the symmetric homogeneous space structure

- A (possibly degenerate) main metric, coming from a suitable rescaling of the Killing-Cartan form.

- When one of the constants $\omega_{1}, \omega_{2}, \ldots, \omega_{m-1}, \omega_{m+1}, \ldots, \omega_{N}$ is equal to zero, then the space $\mathcal{S}^{(m)}$ has a fibered structure. This can be considered as an invariant foliation, each of whose leaves carries a subsidiary metric, coming again from the Killing-Cartan form through restriction to the leaves and suitable rescaling.

- Finally, the canonical connection and the complete hierarchy of subsidiary metrics are compatible.

All the constants $\omega_{1}, \omega_{2}, \ldots, \omega_{N}$ receive a geometrical meaning in the spaces $\mathcal{S}^{(m)}$. The metrics in $\mathcal{S}^{(m)}$ depends on the constants $\omega_{1}, \ldots, \omega_{m-1}, \omega_{m+1}, \ldots, \omega_{N}$. At the tangent space at the origin, the 'main' metric matrix has the diagonal block structure $\operatorname{diag}\left(g^{(m)}, \omega_{m-1} g^{(m)}, \omega_{m-2} \omega_{m-1} g^{(m)}, \ldots, \omega_{1} \ldots \omega_{m-2} \omega_{m-1} g^{(m)}\right)$, where each block $g^{(m)}$ itself is a diagonal matrix $\operatorname{diag}\left(1, \omega_{m+1}, \omega_{m+1} \omega_{m+2}, \ldots, \omega_{m+1} \omega_{m+2} \ldots \omega_{N}\right)$. When all these constants are different from zero, the main metric in $\mathcal{S}^{(m)}$ is nondegenerate; the invariant canonical connection turns out to be the corresponding 
Levi-Civita metric connection, and the value $\omega_{m}$ appears as the sectional curvature of certain plane directions; strictly speaking these spaces are not of constant curvature as this is usually understood for (rank one) Riemannian spaces, yet this structure is as close to constant curvature as a higher rank space can be.

Let us note that within the CK space $\mathcal{S}^{(1)} \equiv S O_{\omega_{1}, \omega_{2}, \ldots, \omega_{N}}(N+1) / S O_{\omega_{2}, \ldots, \omega_{N}}(N)$, the subalgebras $\mathfrak{h}^{(m)}$ are identified with the isotropy subalgebras of a point (for $m=1$ ), of a line (for $m=2$ ), .., of a hyperplane (for $m=N$ ). As $\mathfrak{h}^{(m)}$ is the isotropy subalgebra of the elements of the space $\mathcal{S}^{(m)}$, we get an easy way of visualizing all the CK spaces: $\mathcal{S}^{(2)}$ is the space of all lines in $\mathcal{S}^{(1)}, \mathcal{S}^{(3)}$ is the space of all 2-planes in $\mathcal{S}^{(1)}$, and so on. In terms of the rank 1 space $\mathcal{S}^{(1)}$ the geometrical meaning of the set of $N$ contractions $\Gamma^{(m)}$ is to describe the behaviour of the space around a point, a line, ..., a $(N-1)$-plane (hyperplane). Remember that $\omega_{m}$ is the curvature of the space $\mathcal{S}^{(m)}$; when translated to the space $\mathcal{S}^{(1)}$ the $\omega_{i}$ describe the behaviour of the space around a point $\left(\omega_{1}\right)$, a line $\left(\omega_{2}\right), \ldots$, an hyperplane $\left(\omega_{N}\right)$.

The systematic use of these ideas affords a very clear and complete image to visualize properties of higher rank CK spaces. Indeed the same game can be played not only in $\mathcal{S}^{(1)}$ but in any $\mathcal{S}^{(k)}$ : elements of each space $\mathcal{S}^{(m)}$ appear naturally as certain submanifolds in $\mathcal{S}^{(k)}$. For example, an element (point) in $\mathcal{S}^{(1)}$ appears in the space $\mathcal{S}^{(2)}$ (identified to the manifold of all lines in $\mathcal{S}^{(1)}$ ) as the submanifold of all lines which are incident to the given point. This way, when the elements determined by each of the isotropy subalgebras $\mathfrak{h}^{(m)}(m=1, \ldots, N)$ are realized in the coset space $\mathcal{S}^{(k)}$, they appear as a submanifold whose dimension is given by $(m-k) k$ when $k \leq m$ and by $(k-m)(N+1-k)$ when $m \leq k$.

\subsection{Trigonometry in CK spaces}

The known analogy between hyperbolical and spherical trigonometry is related to the known duality for their homogeneous spaces. The consistent use of the CK scheme allows to push this analogy further on.

Three non collinear points on three lines in a CK space $\mathcal{S}^{(1)}$ of any dimension always lie on a totally geodesic two dimensional submanifold, and therefore the study of their trigonometry reduces to the one of the two-dimensional space $\mathcal{S}^{(1)}$ associated to the three dimensional CK algebra $s \omega_{\omega_{1}, \omega_{2}}(3)$. These nine essentially different spaces are labelled by the two constants $\omega_{1}, \omega_{2}$. The three most known spaces are the two-dimensional Riemannian spaces of constant curvature $\omega_{1}$, (the sphere, the Euclidean plane or the hyperbolical Lobachewski plane, with constants $\left.\omega_{1}, \omega_{2}=1\right)$. The trigonometry of spaces with $\omega_{2} \leq 0$ is less known. For $\omega_{1}=0, \omega_{2}=$ $-1 / c^{2}$, we have the Minkowski $(1+1)$ space, whose trigonometry was discussed by Birman and Nomizu $\mathrm{Q}$. The basic trigonometric formulas for these nine spaces are given, without proof, in the book by Yaglom 1 . We show here how to formulate trigonometry for all nine CK 2-d spaces in one stroke. Let us consider a pure triangle in $\mathcal{S}^{(1)}$ : three points which determine three lines (as a model, think of a pure triangle as a triangle with time-like sides in Minkowski space). Then the sides $a, b, c$ and the angles $A, B, C$ (labelled as usual, and with $a$ the largest side) can be defined as the 
values of the canonical parameters in the one-parameter family of translations along one side (rotations around a vertex), which carry one vertex to the other vertex on the side (one side to the other side through the vertex). This definition coincides with the one got by using the geodesic distances asociated to the canonical metrics in the spaces of points and of lines. A striking peculiarity which follows from this approach should be remarked: the larger angle $A$ corresponds to the external angle in Euclid. Let us call $P_{a}, P_{b}, P_{c}$ the generators of translations along the three sides (all three generators conjugated to the basic translation generator $P_{1}:=\Omega_{01}$ in the CK algebra), and $J_{A}, J_{B}, J_{C}$ the generators of rotations around the three vertices (all three conjugated to the basic rotation generator $J:=\Omega_{12}$ ).

All equations of trigonometry can be derived from the two conditions:

- $\exp \left(b P_{b}\right) \exp \left(-a P_{a}\right) \exp \left(c P_{c}\right)$ must be a rotation around vertex $A$

- $\exp \left(B J_{B}\right) \exp \left(-A J_{A}\right) \exp \left(C J_{C}\right)$ must be a traslation along side $a$

These requirement leads to a complete set of equations relating sides and angles, which can be adequately written in terms of the generalised cosine $\mathrm{C}_{\omega}(x)$, sine $\mathrm{S}_{\omega}(x)$, and versed sine $\mathrm{V}_{\omega}(x)$ functions, which depend on a label and reduce to either circular or hyperbolic functions when the label is 1 or -1 and to the functions $1, x$ and $x^{2} / 2$ when the label $\omega=0$. All spherical, euclidean and hyperbolic trigonometry can be shown to be the particular specialization of these general equations for the cases where the constant $\omega_{2}=1$, while $\omega_{1}$ is either positive, zero or negative 10 . The basic relations are:

$$
\begin{aligned}
& \mathrm{S}_{\omega_{1}}(a) / \mathrm{S}_{\omega_{2}}(A)=\mathrm{S}_{\omega_{1}}(b) / \mathrm{S}_{\omega_{2}}(B)=\mathrm{S}_{\omega_{1}}(c) / \mathrm{S}_{\omega_{2}}(C), \\
& \mathrm{V}_{\omega_{1}}(a)=\mathrm{V}_{\omega_{1}}(b+c)-\omega_{2} \mathrm{~V}_{\omega_{2}}(a) \mathrm{S}_{\omega_{1}}(b) \mathrm{S}_{\omega_{1}}(c), \\
& \mathrm{V}_{\omega_{2}}(A)=\mathrm{V}_{\omega_{2}}(B+C)-\omega_{1} \mathrm{~V}_{\omega_{1}}(a) \mathrm{S}_{\omega_{2}}(B) \mathrm{S}_{\omega_{2}}(C),
\end{aligned}
$$

where one can recognize the general form of the sine, cosine and dual cosine theorems, the two last in their versed sine form. Another remarkable consequence we get using this approach are the identities

$$
\begin{aligned}
& \exp \left(b P_{b}\right) \exp \left(-a P_{a}\right) \exp \left(c P_{c}\right)=\exp \left((B-A+C) J_{A}\right), \\
& \exp \left(B J_{B}\right) \exp \left(-A J_{A}\right) \exp \left(C J_{C}\right)=\exp \left((b-a+c) P_{a}\right),
\end{aligned}
$$

which give the holonomies associated to the triangle, both in the space of points and in the space of lines.

The ordinary duality $\omega_{1} \rightarrow-\omega_{1}$ is automatically contained in the fact that all sides appear in functions with the label $\omega_{1}$; similarly there is another duality for angles and $\omega_{2}$. However the most interesting property in this approach is the complete invariance under the simultaneous interchange sides $\leftrightarrow$ angles and $\omega_{1} \leftrightarrow \omega_{2}$.

When both constants $\omega_{1}, \omega_{2} \rightarrow 0$, these equations reduce to:

$$
\frac{a}{A}=\frac{b}{B}=\frac{c}{C}, \quad A^{2}=(B+C)^{2}, \quad a^{2}=(b+c)^{2},
$$

which for the kinematics of the $(1+1)$ Galilean space time are elementary physical properties (in particular, the two last equations are but the velocity addition law and the absolute character of time). 
A possibility opened by this approach is to consider the trigonometrical equations for all CK spaces, and to look how these equations depend on the two curvatures $\omega_{1}, \omega_{2}$. For the 'fiducial' case $\omega_{1}=\omega_{2}=0$ the basic equations are simple, linear ones. One may look to the general equations as some classical deformations of these purely linear ones (eq. 9). For any equation the corresponding 'deformation' involves two stages: Firstly, sides and angles are replaced everywhere they appear by a trigonometrical function whose label is the appropiate curvature: $a \rightarrow \mathrm{S}_{\omega_{1}}(a), a^{2} \rightarrow \mathrm{V}_{\omega_{1}}(a), A \rightarrow \mathrm{S}_{\omega_{2}}(A), A^{2} \rightarrow \mathrm{V}_{\omega_{2}}(A)$, etc. and secondly, eventual additional terms involving explicitly the curvatures $\omega_{1}, \omega_{2}$ may appear. It is however posible to write all equations in some equivalent 'minimal' way, where curvatures $\omega_{1}, \omega_{2}$ do not appear explicitly, sides and angles appear always through their sines, $\mathrm{S}_{\omega_{1}}(a), \mathrm{S}_{\omega_{2}}(A)$, and further to that, the deformation is described by some 'missing' cosines. We say here 'missing' as when $\omega=0$, all cosines equal identically to 1 , so that they are simply invisible. These ideas are worth of further development.

The approach to trigonometry we have sketched here has been already developped for hermitian CK spaces, and should also be extended to the scarcely known trigonometry of higher rank spaces.

\section{Acknowledgements}

M.S. would like to acknowledge the organisers of the II IWCQIS for their invitation and hospitality during the meeting. This work has been partially supported by a DGICYT project (PB94-1115) from Ministerio de Educación y Ciencia de España.

\section{References}

1. D. M. Y. Sommerville, Classification of geometries with projective metrics, Proc. Edinburgh. Math. Soc., 28, (1910-11), 25. I. M. Yaglom, B. A. Rozenfel'd and E. U. Yasinskaya, Projective metrics, Sov Math. Surveys, 19, (1966), 49.

2. B. A. Rozenfel'd, A history of non-euclidean geometry (Springer, New York, 1988)

3. L. M. Nieto, J. Negro and M. Santander, Two Dimensional Cayley-Klein algebras generated by Expansions, this volume.

4. F. J. Herranz, M. de Montigny, M. A. del Olmo and M. Santander, J. Phys. A: Math. Gen. 27, (1994), 2515. F. J. Herranz and M. Santander, J. Phys. A: Math. Gen. 29, (1996), 6643.

5. J. A de Azcárraga, F. J. Herranz, J. C. Pérez Bueno and M. Santander, A general analysis of the cohomology of $s o(N+1)$ contractions, forthcoming

6. F. J. Herranz and M. Santander, The Casimirs invariants for quasi-orthogonal algebras and their application to expansions, forthcoming

7. G. S. Birman and K. Nomizu, Amer. Math. Month., 91, (1984), 543.

8. I. M. Yaglom, A simple non-euclidean geometry and its physical basis, (Springer, New York, 1979)

9. A. Ballesteros, F. J. Herranz, M. A. del Olmo and M. Santander J. Phys. A: Math. Gen., 26, (1993), 5801.

10. F. J. Herranz, PhD Thesis, Universidad de Valladolid, 1995 